\title{
ERRATUM
}

http://dx.doi.org/10.1007/s00376-018-0001-7

\section{Erratum to: The Quadrennial Ozone Symposium 2016}

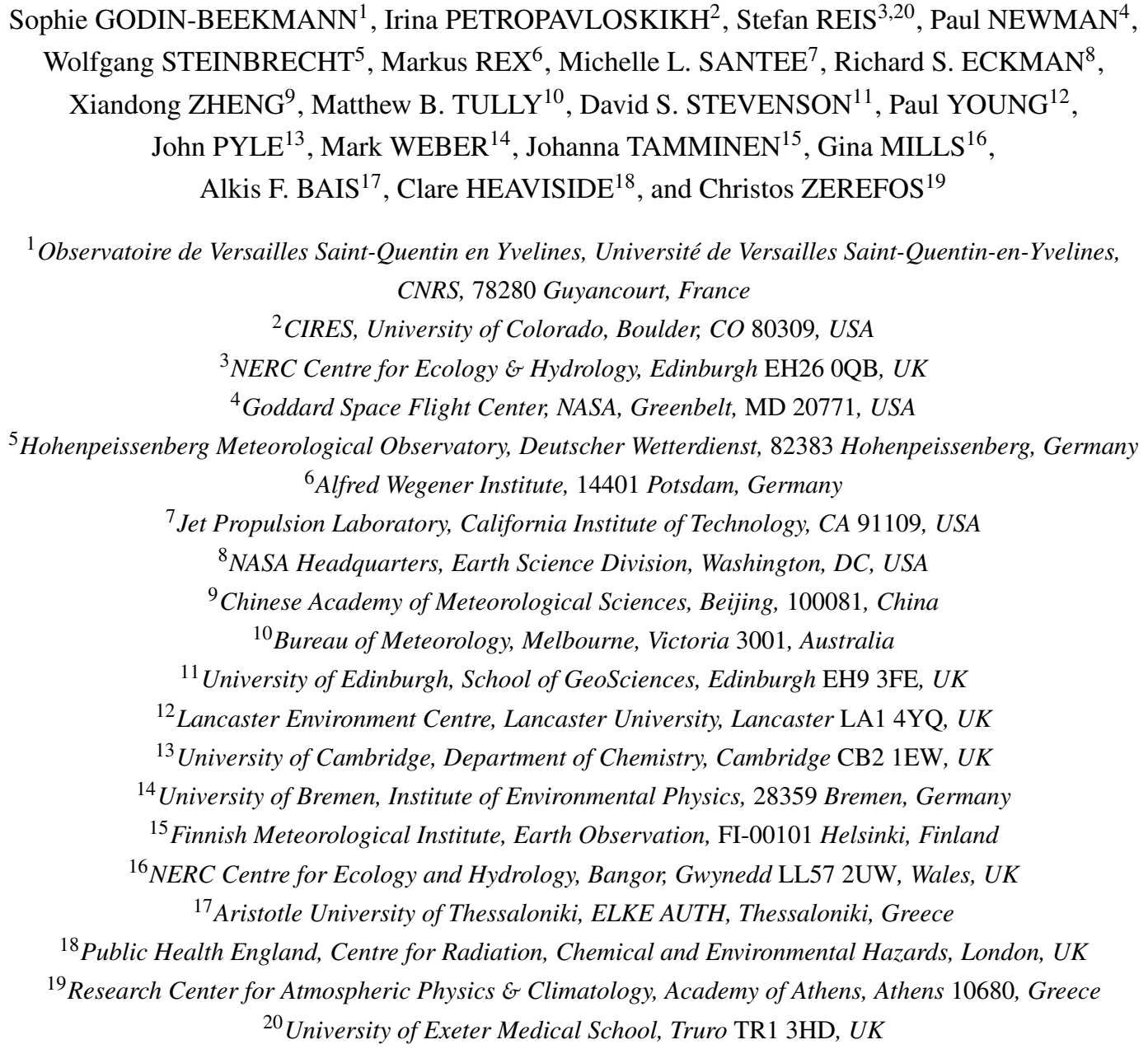

Erratum to: Godin-Beekmann, S., and Coauthors, 2017: The Quadrennial Ozone Symposium 2016. Adv. Atmos. Sci., 34(3), 368-369, https://doi.org/10.1007/s00376-016-6309-2.

In the original version of this article, some authors' names were misspelled. The correct version is as follows: 


\title{
The Quadrennial Ozone Symposium 2016
}

\author{
Sophie GODIN-BEEKMANN*1, Irina PETROPAVLOVSKIKH ${ }^{2}$, Stefan REIS ${ }^{3,20}$, Paul NEWMAN ${ }^{4}$, \\ Wolfgang STEINBRECHT ${ }^{5}$, Markus REX ${ }^{6}$, Michelle L. SANTEE ${ }^{7}$, Richard S. ECKMAN ${ }^{8}$, \\ Xiangdong ZHENG ${ }^{9}$, Matthew B. TULLY ${ }^{10}$, David S. STEVENSON ${ }^{11}$, Paul YOUNG ${ }^{12}$, \\ John PYLE ${ }^{13}$, Mark WEBER ${ }^{14}$, Johanna TAMMINEN ${ }^{15}$, Gina MILLS ${ }^{16}$, Alkiviadis F. BAIS ${ }^{17}$, \\ Clare HEAVISIDE $^{18}$, and Christos ZEREFOS ${ }^{19}$ \\ ${ }^{1}$ Observatoire de Versailles Saint-Quentin en Yvelines, Université de Versailles Saint-Quentin-en-Yvelines, \\ CNRS, 78280 Guyancourt, France \\ ${ }^{2}$ CIRES, University of Colorado, Boulder, CO 80309, USA \\ ${ }^{3}$ NERC Centre for Ecology $\mathcal{G}$ Hydrology, Edinburgh EH26 0QB, UK \\ ${ }^{4}$ Goddard Space Flight Center, NASA, Greenbelt, MD 20771, USA \\ ${ }^{5}$ Hohenpeissenberg Meteorological Observatory, Deutscher Wetterdienst, 82383 Hohenpeissenberg, Germany \\ ${ }^{6}$ Alfred Wegener Institute, 14401 Potsdam, Germany \\ ${ }^{7}$ Jet Propulsion Laboratory, California Institute of Technology, CA 91109, USA \\ ${ }^{8}$ NASA Headquarters, Earth Science Division, Washington, DC, USA \\ ${ }^{9}$ Chinese Academy of Meteorological Sciences, Beijing, 100081, China \\ ${ }^{10}$ Bureau of Meteorology, Melbourne, Victoria 3001, Australia \\ ${ }^{11}$ University of Edinburgh, School of GeoSciences, Edinburgh EH9 3FE, UK \\ ${ }^{12}$ Lancaster Environment Centre, Lancaster University, Lancaster LA1 4YQ, UK \\ ${ }^{13}$ University of Cambridge, Department of Chemistry, Cambridge CB2 1EW, UK \\ ${ }^{14}$ University of Bremen, Institute of Environmental Physics, 28359 Bremen, Germany \\ ${ }^{15}$ Finnish Meteorological Institute, Earth Observation, FI-00101 Helsinki, Finland \\ ${ }^{16}$ NERC Centre for Ecology and Hydrology, Bangor, Gwynedd LL57 2UW, Wales, UK \\ ${ }^{17}$ Aristotle University of Thessaloniki, Thessaloniki, Greece \\ ${ }^{18}$ Public Health England, Centre for Radiation, Chemical and Environmental Hazards, London, UK \\ ${ }^{19}$ Research Center for Atmospheric Physics $\mathcal{E}$ Climatology, Academy of Athens, Athens 10680, Greece \\ ${ }^{20}$ University of Exeter Medical School, Truro TR1 3HD, UK
}

The online version of this original article can be found at http://dx.doi.org/10.1007/s00376-016-6309-2

\footnotetext{
* Corresponding author: Sophie GODIN-BEEKMANN

Email: sophie.godin-beekmann@latmos.ipsl.fr
} 